Case Report

\title{
Extensive Bilateral Intracranial Calcifications: A Case of Iatrogenic Hypoparathyroidism
}

\author{
Vaso Zisimopoulou, ${ }^{1}$ Anna Siatouni, ${ }^{2}$ Grigorios Tsoukalos, ${ }^{3}$ \\ Antonios Tavernarakis, ${ }^{1}$ and Stylianos Gatzonis ${ }^{2}$ \\ ${ }^{1}$ Department of Neurology, Evaggelismos Hospital, 45-47 Ipsilantou Street, 10676 Athens, Greece \\ ${ }^{2}$ Department of Neurosurgery, Athens Medical School, Evaggelismos Hospital, 45-47 Ipsilantou Street, 10676 Athens, Greece \\ ${ }^{3}$ Department of Radiology, Evaggelismos Hospital, 45-47 Ipsilantou Street, 10676 Athens, Greece
}

Correspondence should be addressed to Vaso Zisimopoulou; vzisimopoulou@gmail.com

Received 12 December 2012; Revised 10 January 2013; Accepted 24 January 2013

Academic Editor: Thomas Vogl

Copyright (C) 2013 Vaso Zisimopoulou et al. This is an open access article distributed under the Creative Commons Attribution License, which permits unrestricted use, distribution, and reproduction in any medium, provided the original work is properly cited.

This is a case of a 69-year-old male patient with long-standing iatrogenic hypoparathyroidism after total thyroidectomy. The clinical evaluation revealed mild neurological symptoms and excessive brain calcinosis. Intracranial calcification that affects structures other than the basal ganglia and the cerebellum is a rare manifestation of postoperative hypoparathyroidism. Detection of brain calcinosis in patients who had total thyroidectomy can motivate clinicians in further investigation of possible hypoparathyroidism with measurement of calcium and phosphorus serum levels.

\section{Introduction}

Intracranial calcification that affects structures other than the basal ganglia and the cerebellum is a rare manifestation of postoperative hypoparathyroidism. The incidence of hypoparathyroidism following total thyroidectomy is reported in several series between $0.3 \%$ and $6.3 \%$ for permanent hypoparathyroidism and between $5 \%$ and $22 \%$ for transient hypoparathyroidism [1]. We present a case of long-standing iatrogenic hypoparathyroidism with bilateral extensive intracranial calcifications and only mild clinical symptoms.

\section{Case Report}

A 69-year-old man presented to the outpatient clinic 48 hours after an episode of loss of consciousness lasting a few seconds. He had a medical history of hypertension and hyperlipidaemia well controlled with medicine and a history of total thyroidectomy 18 years ago treated since then with levothyroxine. The patient's clinical course after surgery is unknown, as he rejected any postoperative followup, and the effort made to obtain any past medical records was ineffective.
Nevertheless, the patient did report exophthalmos, excessive weight loss, and a large goitre before surgery, as well as calcium supplementation for a month after surgery, which he stopped taking by himself. Following the aforementioned patient's statements, Grave's disease was presumed to be the indication for total thyroidectomy. Neurological examination showed only mild extrapyramidal signs (mild rigidity and bradykinesia but not rest tremor) and the presence of more than one primitive reflex. A brain computed tomography (CT) scan revealed extensive bilateral symmetrical brain calcifications in the frontal lobes, basal ganglia, subcortical and periventricular white matter, and in the cerebellar hemispheres (Figures 1, 2, and 3). Minimental scale examination revealed a score of 24/30 (noted that the patient had only primary education). Family members reported no profound signs of dementia or incapability throughout patient's everyday life. The patient himself reported fatigability and a tingling sensation around the mouth. On investigation, his calcium level was $5.3 \mathrm{mg} / \mathrm{dL}$ (normal values (nv) 8.5$10.5 \mathrm{mg} / \mathrm{dL}$ ), serum albumin $4.0 \mathrm{~g} / \mathrm{dL}$ (nv $3.5-5 \mathrm{~g} / \mathrm{dL}$ ), serum magnesium $2.22 \mathrm{mg} / \mathrm{dL}$ (nv $1.58-2.55 \mathrm{mg} / \mathrm{dL}$ ), serum phosphate $5.4 \mathrm{mg} / \mathrm{dL}$ ( $\mathrm{nv} 2.5-5 \mathrm{mg} / \mathrm{dL}$ ), PTH was undetectable, and TSH was normal $2.2 \mu \mathrm{U} / \mathrm{mL}$ (nv $0.27-4.2 \mu \mathrm{U} / \mathrm{mL}$ ). The 


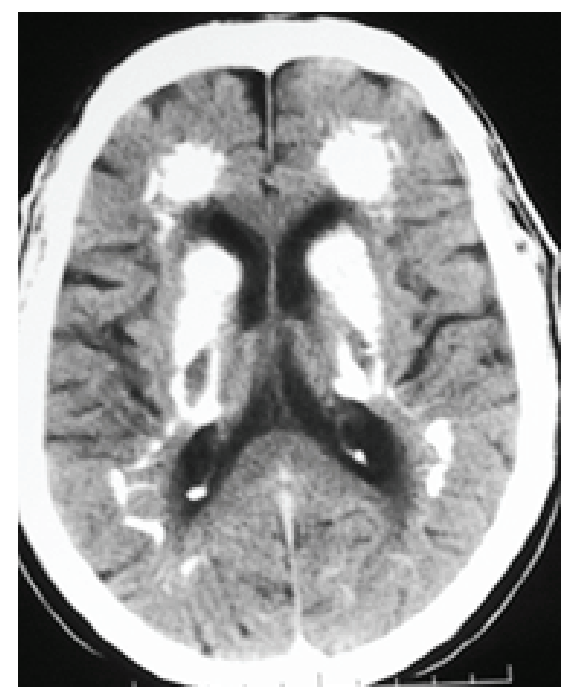

FIGURE 1: CT scan axial view demonstrating extensive bilateral calcifications in the periventricular white matter (frontal horns, basal ganglia, and internal capsule).

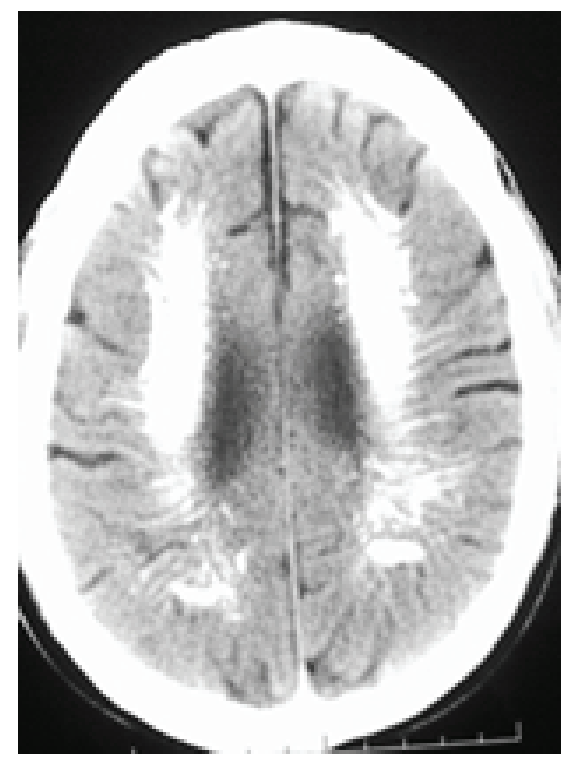

FIGURE 2: CT scan axial view demonstrating extensive bilateral calcifications in the periventricular white matter, semiovale center, and corona radiate.

rest of the laboratory evaluation was unremarkable. An EEG showed evolution of alpha dominance and rare slow wave bursts of theta and delta activity. The clinical presentation of our patient was attributed to hypoparathyroidism. To prevent further complications from hypoparathyroidism, patient was prescribed with calcitriol $0.5 \mu \mathrm{g} /$ day and calcium $1 \mathrm{gm} /$ day. With normalization of serum calcium levels $(8.7 \mathrm{mg} / \mathrm{dL})$, patient had improvement of extrapyramidal signs and complete diminish of paresthesias. Patient was followed up for a year, and he remained in good mental and physical health.

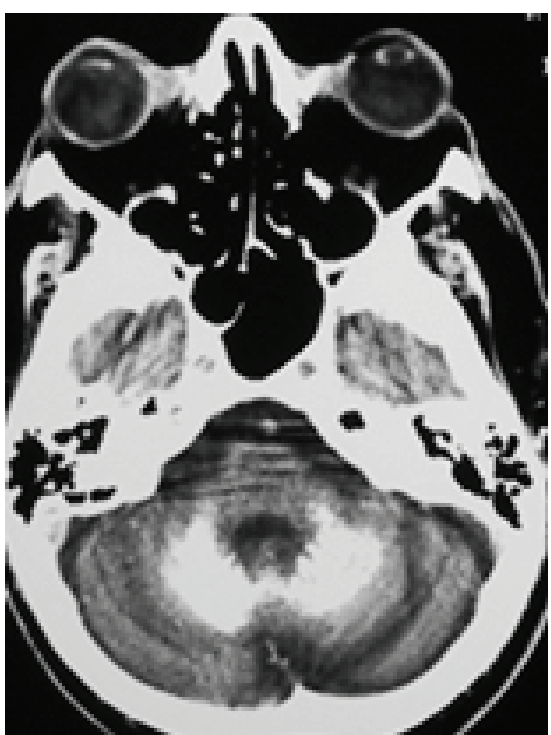

FIgURE 3: CT scan axial view demonstrating calcifications in both cerebellar hemispheres.

\section{Discussion}

Postoperative hypoparathyroidism is the most common complication of complete or near-complete extirpation of thyroid gland, by destruction or vascular compromise of parathyroid tissue [2]. Several thyroid conditions such as Grave's disease, thyrotoxicosis as a result of hyperactive thyroid adenomas, recurrent goiter, and thyroid carcinoma carry a higher risk to develop transient and permanent hypoparathyroidism postoperatively [3]. The main clinical features of hypoparathyroidism are a result of induced hypocalcaemia and can range from a life threatening condition to an asymptomatic laboratory finding [4]. Hypocalcaemia most commonly presents with paresthesia, cramps, muscle spasms, circumoral numbness, and seizures but can also present with laryngospasm, neuromuscular irritability, cognitive impairment, personality disturbances, prolonged QT intervals, electrocardiographic changes that mimic myocardial infarction, or heart failure [5].

Intracranial calcification is one of the features of chronic hypocalcaemia, and the calcifications typically involve the basal ganglia, thalami, and the cerebellum [6]. In our patient, calcinosis exceeds the common brain locations and involves the subcortical white matter of the frontal and parietal lobes. A review of the literature reveals only few case reports of excessive calcification of subcortical white matter regarding postoperative hypoparathyroidism [7-10]. The most commonly reported manifestations of postoperative hypoparathyroidism with basal ganglia calcification are parkinsonism [11] and seizures [12-14]. There are also reports of cognitive impairment [15] and even intracerebral hemorrhage [16]. The remarkable point of our case is the discordance between imaging and clinical symptoms and signs. Despite the wide brain calcification, the patient had only mild symptoms and signs.

The pathogenic mechanism of brain calcinosis in postoperative hypoparathyroidism is not yet defined. Although 
Virchow [17] and Bamberger and Von Rokitansky [18] independently described the histology of bilateral basal ganglia calcifications in 1855, it was not until 1939 that their association with chronic hypoparathyroidism was recognized by Eaton et al. [19]. Microscopic colloid deposition around cerebral blood vessels is followed by calcification most commonly in the basal ganglia [20]. According to Goswami et al. the progression of basal ganglia calcification is related to the calcium/phosphorus ratio [21]; thus, a strict control of hypocalcaemia and hypophosphatemia upon diagnosis is mandatory.

\section{Conclusion}

Chronic hypocalcaemia due to postoperative hypoparathyroidism can remain subclinical for long and detection of intracranial calcifications can be the trigger for further investigation with measurement of calcium and phosphorus levels. Calcium supplementation protects the patient from further complications of chronic hypoparathyroidism.

\section{References}

[1] P. Miccoli, M. N. Minuto, and M. Miccoli, "Incidence of morbidity following thyroid surgery," in Thyroid Surgery: Preventing and Managing Complications, P. Miccoli, D. J. Terris, M. N. Minuto, and M. W. Seybt, Eds., pp. 6-7, John Wiley \& Sons, Ltd, Oxford, UK, 2012.

[2] Wysolmerski, J. John, and L. K. Insogna, "The parathyroid glands, hypercalcemia, and hypocalcemia," in Goldman's Cecil Medicine, L. R. Cecil, L. Goldman, and I. A. Schafer, Eds., p. 1597, Elsevier/Saunders, Philadelphia, Pa, USA, 2011.

[3] O. Thomusch, A. Machens, C. Sekulla, J. Ukkat, M. Brauckhoff, and H. Dralle, "The impact of surgical technique on postoperative hypoparathyroidism in bilateral thyroid surgery: A multivariate analysis of 5846 consecutive patients," Surgery, vol. 133, no. 2, pp. 180-185, 2003.

[4] M. S. Cooper and N. J. Gittoes, "Diagnosis and management of hypocalcaemia," British Medical Journal, vol. 336, no. 7656, pp. 1298-1302, 2008.

[5] J. Fong and A. Khan, "Hypocalcemia: updates in diagnosis and management for primary care," Canadian Family Physician, vol. 58, no. 2, pp. 158-162, 2012.

[6] M. Karimi, F. Habibzadeh, and V. De Sanctis, "Hypoparathyroidism with extensive intracerebral calcification in patients with $\beta$-thalassemia major," Journal of Pediatric Endocrinology and Metabolism, vol. 16, no. 6, pp. 883-886, 2003.

[7] J. H. Friedman, J. Chiucchini, and J. R. Tucci, "Idiopathic hypoparathyroidism with extensive brain calcification and persistent neurologic dysfunction," Neurology, vol. 37, no. 2, pp. 307-309, 1987.

[8] C. Lang, W. Huk, and J. Pichl, "Comparison of extensive brain calcification in postoperative hypoparathyrodism on CT and NMR scan," Neuroradiology, vol. 31, no. 1, pp. 29-32, 1989.

[9] Y. Litvin, A. Rosler, and R. A. Bloom, "Extensive cerebral calcification in hypoparathyroidism," Neuroradiology, vol. 21, no. 5, pp. 271-272, 1981.

[10] P. G. Jorens, B. J. Appel, F. A. Hilte, C. Mahler, and P. P. De Deyn, "Basal ganglia calcifications in postoperative hypoparathyroidism: A case with unusual characteristics," Acta Neurologica Scandinavica, vol. 83, no. 2, pp. 137-140, 1991.
[11] S. Abe, K. Tojo, K. Ichida et al., "A rare case of idiopathic hypoparathyroidism with varied neurological manifestations," Internal Medicine, vol. 35, no. 2, pp. 129-134, 1996.

[12] C. R. Basak, "A case report of Basal Ganglia calcification-a rare finding of hypoparathyroidism," Oman Medical Journal, vol. 24, no. 3, pp. 220-222, 2009.

[13] S. T. Reddy and R. D. Merrick, "Hypoparathyroidism, intracranial calcification, and seizures 61 years after thyroid surgery," Tennessee Medicine, vol. 92, no. 9, pp. 341-342, 1999.

[14] I. Rizvi, N. A. Ansari, M. Beg, and M. D. Shamim, "Widespread intracranial calcification, seizures and extrapyramidal manifestations in a case of hypoparathyroidism," North American Journal of Medical Sciences, vol. 4, no. 8, pp. 369-372, 2012.

[15] L. Gionanlis, A. Vainas, G. Bamihas, P. Veneti, and K. Sobolos, "Brain calcinosis in a dialysis patient with hypoparathyroidism," NDT Plus, vol. 1, no. 1, pp. 36-40, 2008.

[16] N. Mamdani, A. L. Repp, B. Seyoum, and P. Berhanu, "Idiopathic hypoparathyroidism presenting with severe hypocalcemia and asymptomatic basal ganglia calcification followed by acute intracerebral bleed," Endocrine Practice, vol. 13, no. 5, pp. 487-492, 2007.

[17] R. Virchow, "Kalk-metastasen," Archiv für Pathologische Anatomie und Physiologie und für Klinische Medicin, vol. 8, no. 1, pp. 103-113, 1855.

[18] Bamberger and K. Von Rokitansky, Lehrbuch der Pathologischen Anatomie, vol. 2, Wilhelm Braumuller, Vienna, Austria, 1856.

[19] L. M. Eaton, J. D. Camp, and J. G. Love, "Symmetric cerebral calcification, particularly of the basal ganglia, demonstrable roentgenographically, calcification of the finer cerebral blood vessels," Archives of Neurology \& Psychiatry, vol. 41, no. 5, Article ID 022701, pp. 921-942, 1939.

[20] J. C. Bennett, R. H. Maffly, and H. L. Steinbach, "The significance of bilateral basal ganglia calcification," Radiology, vol. 72, no. 3, pp. 368-378, 1959.

[21] R. Goswami, R. Sharma, V. Sreenivas, N. Gupta, A. Ganapathy, and S. Das, "Prevalence and progression of basal ganglia calcification and its pathogenic mechanism in patients with idiopathic hypoparathyroidism," Clinical Endocrinology, vol. 77, no. 2, pp. 200-206, 2012. 


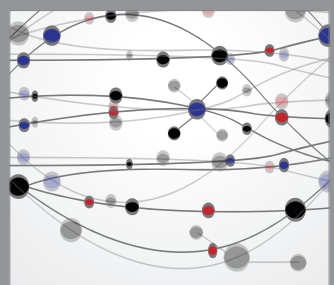

The Scientific World Journal
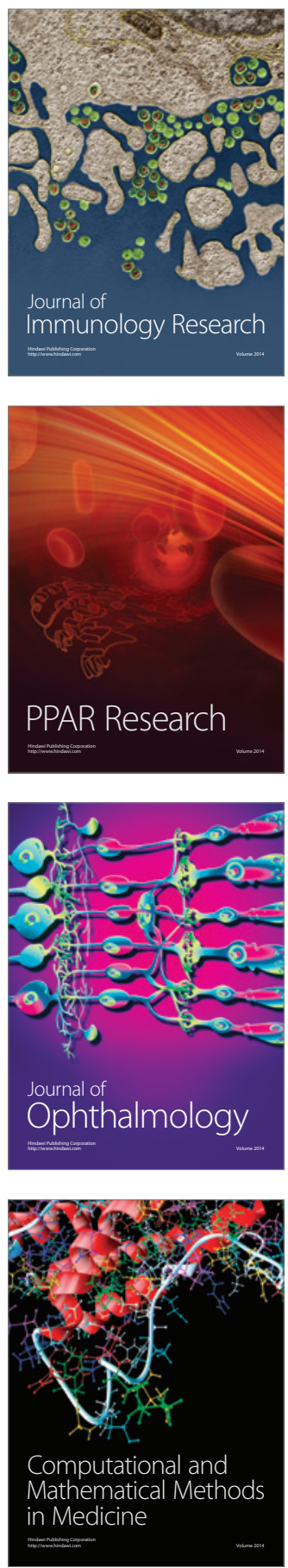

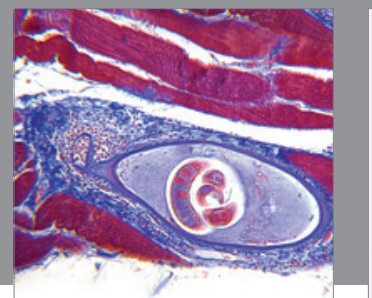

Gastroenterology

Research and Practice
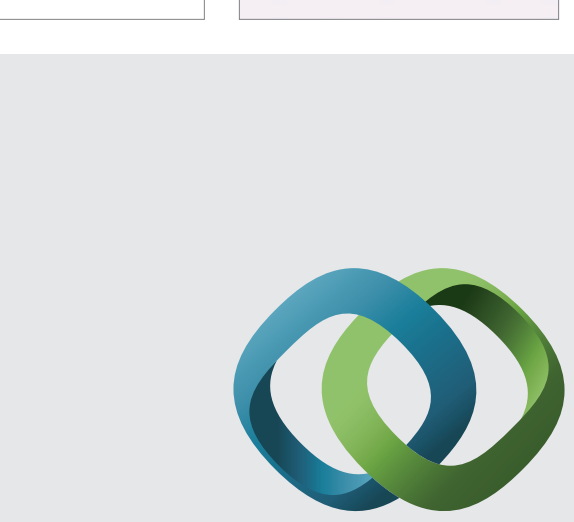

\section{Hindawi}

Submit your manuscripts at

http://www.hindawi.com
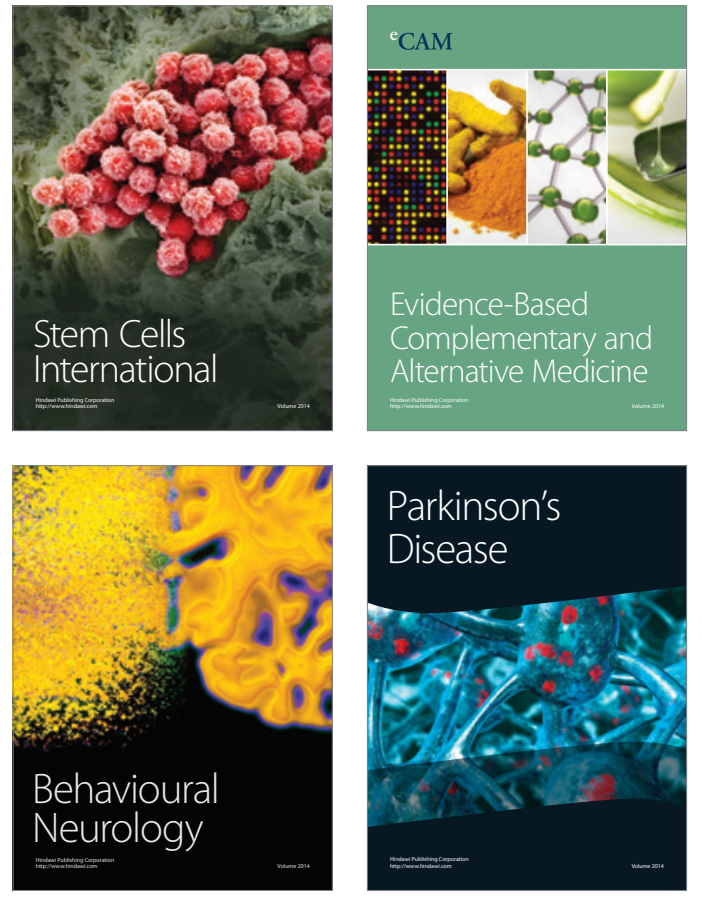
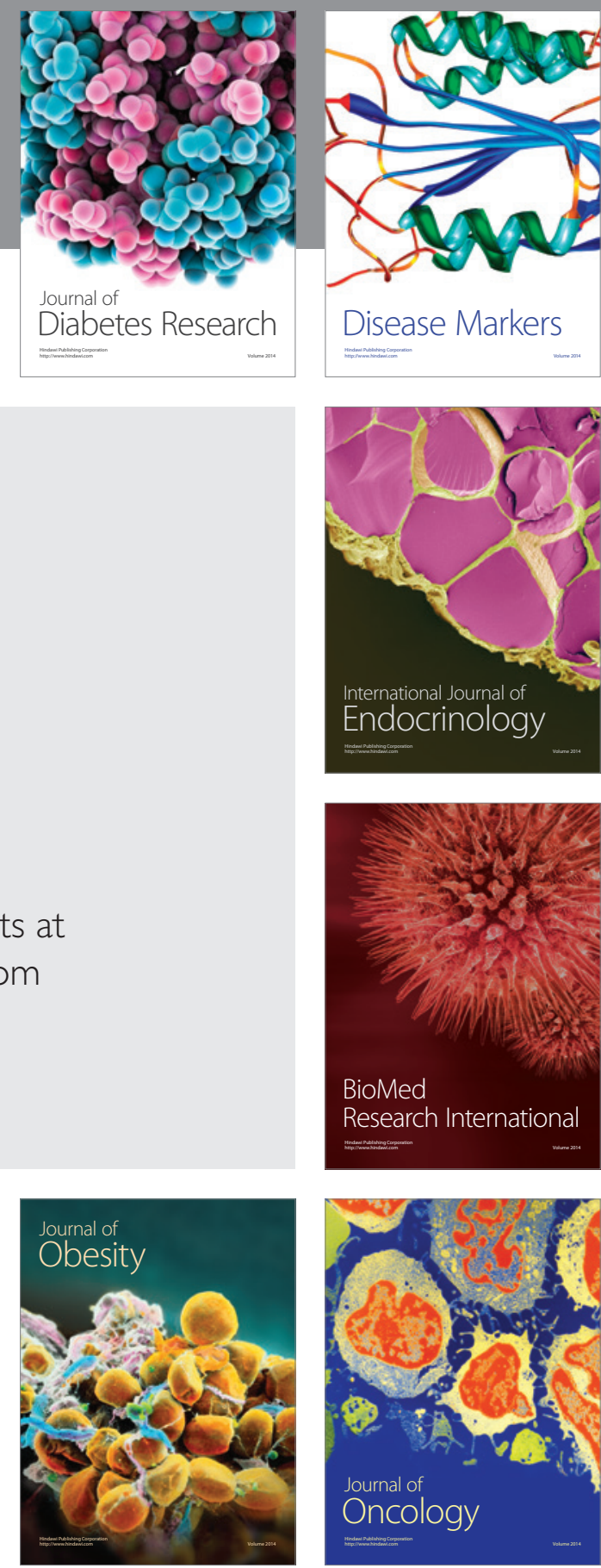

Disease Markers
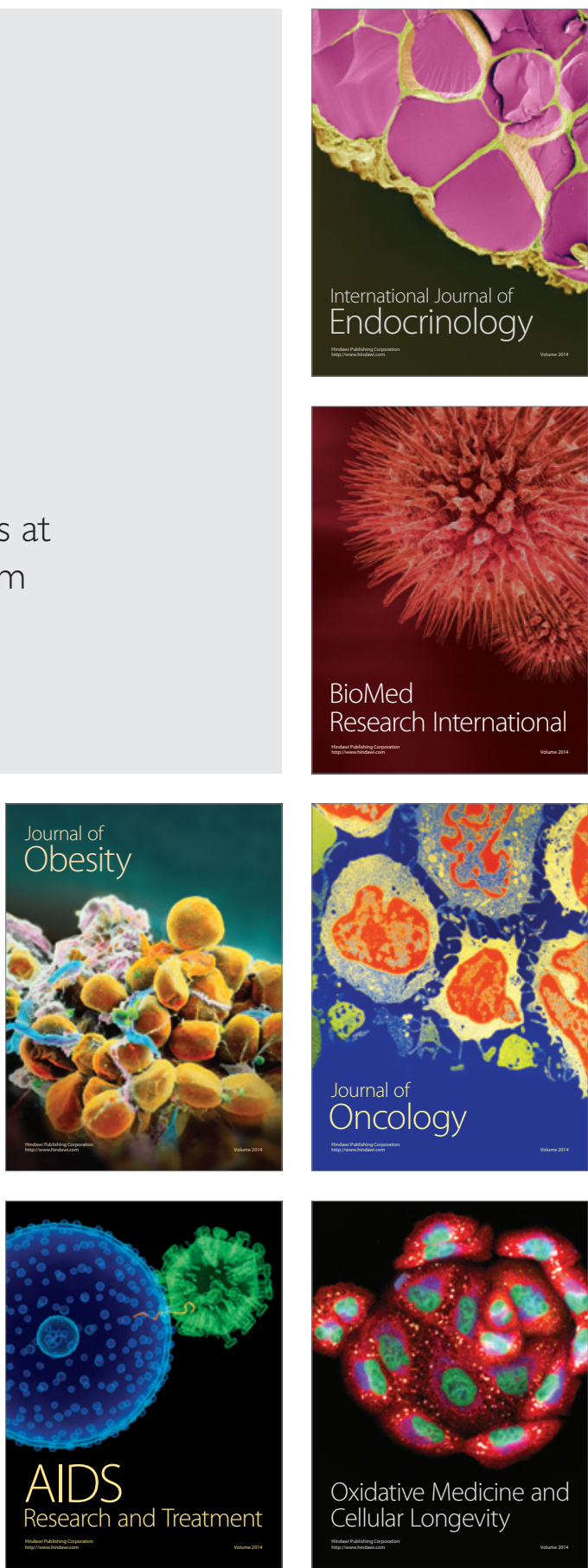